\title{
Comparison of inhaled versus intravenous anesthesia for laryngoscopy and laryngeal electromyography in a rat model
}

\author{
M. Gazzaz ${ }^{1 *}$ (D) J. Saini ${ }^{2,3}$, S. Pagliardini ${ }^{2,3,4}$, B. Tsuil ${ }^{5}$, C. Jeffery ${ }^{1}$ and H. El-Hakim ${ }^{1}$
}

\begin{abstract}
Background: Propofol and remifentanil intravenous combination is one popular form of total intravenous anesthesia (TIVA) in mainstream clinical practice, but it has rarely been applied to a rat model for laryngoscopy and laryngeal electromyography (LEMG). Our objective was to establish a safe and reproducible general anesthetic protocol for laryngoscopy and endoscopic LEMG in a rat model. Our hypothesis is that TIVA allows a minimally morbid, and feasible laryngoscopy and LEMG.

Methods: Sprague Dawley rats were subjected to either inhalational anesthesia (IA) (isoflurane) or TIVA (propofol and remifentanil) and underwent laryngoscopy and LEMG. The primary outcome was a complete minimally interrupted rigid laryngoscopy and obtaining reproducible motor unit potentials from the posterior cricoarytenoid muscles. The secondary outcome was morbidity and mortality.

Results: Seventeen out of twenty-two rats underwent both TIVA and IA. Only two underwent IA only. All nineteen rats that underwent IA had a successful experiment. Seventeen rats underwent TIVA, however, only nine completed a successful experiment due to difficulty achieving a surgical plane, and respiratory events. Upon comparing the success of the two anaesthetic regimens, IA was superior to TIVA $(P=0.0008)$. There was no statistical difference between the amplitudes ( $p=0.1985)$ or motor units burst duration $(p=0.82605)$ of both methods. Three mortalities were encountered, one of which was due to lidocaine toxicity and two were during anesthetic induction. Respiratory related morbidity was encountered in two rats, all seen with TIVA.

Conclusions: TIVA is not an ideal anesthetic regimen for laryngeal endoscopy and LEMG in rat models. Contrary to our hypothesis, IA did not affect the quality of the LEMG and allowed a seamless rigid endoscopy.
\end{abstract}

Keywords: Laryngeal mobility disorders, Laryngeal electromyography, Inhalational anesthesia, Total intravenous anesthesia

\section{Background}

The standards of general anesthesia for airway endoscopy in humans have evolved due to developing technological and pharmacological innovations. One of the most challenging diagnoses to establish in laryngology is mobility disorders, particularly in children where endoscopy under

\footnotetext{
* Correspondence: mgazzaz@ualberta.ca

This work was presented on the podium at the Poliquin Residents Research Competition (Canadian Society of Otolaryngology-Head and Neck Surgery Annual Meeting, June 2018, Quebec City, QC).

'Division of Otolaryngology-Head and Neck Surgery, Department of Surgery, University of Alberta, 2C3.57 Walter MacKenzie Centre, Edmonton, AB T6G 2R7, Canada

Full list of author information is available at the end of the article
}

general anesthesia, supplemented accordingly by laryngeal electromyography (LEMG) is the reference standard.

Traditionally, inhalational anesthesia (IA) was routinely employed for airway endoscopy in clinical practice. In the late 1990's, total intravenous anesthesia (TIVA) technique was introduced and gained popularity [1]. But to this day, proponents of both options argue their cases strongly. The claimed advantages of IA include speed, ease and comfortable induction using a mask in the absence of intravenous (IV) access, in addition to simple non-invasive evaluation of the blood tension of the inhaled agent [2]. On the other hand, TIVA is professed to lessen postoperative nausea and vomiting, act rapidly and independently from the alveolar ventilation, and is

(c) The Author(s). 2018 Open Access This article is distributed under the terms of the Creative Commons Attribution 4.0 International License (http://creativecommons.org/licenses/by/4.0/), which permits unrestricted use, distribution, and 
administrable using peripheral locations away from airway instrumentation. It is also a non-pollutant for the operative room environment [2-4].

Some experts in airway endoscopy and electromyography studies support the notion that anesthetic agents may modify the findings. It is proposed that different concentration and duration of IA may modify LEMG findings and produce spurious abnormalities. Some rest this notion on evidence from literature pertaining to spine surgery [5-8]. However, many centers perform LEMG in humans under IA [9-12], especially that there are no head to head studies comparing the two techniques. In rats, LEMG has also been performed under IA [13], yet in most cases it has been used as an induction agent for sedation [14-17].

Propofol and remifentanil IV combination is one popular form of TIVA in mainstream clinical practice, but it has rarely been applied to a rat model for laryngoscopy and LEMG. We therefore set out to evaluate whether a TIVA protocol is applicable to the rat model for reproducible assessment of laryngeal function, with minimal morbidity. We specifically aimed to compare the mortality, morbidity, and reproducibility of two general anesthetic protocols for laryngoscopy and endoscopic LEMG in a rat model. Our hypothesis was that TIVA allows a minimally morbid, and feasible laryngoscopy and LEMG.

\section{Methods}

\section{Study design}

The experiment was conducted in accordance with the Canadian Council of Animal Care guidelines and policies, following approval from the University of Alberta Health Research Ethics Board (AUP00001311) and the Animal Care and Use Committee for health sciences at the University of Alberta.

This prospective comparative non-randomized, cross over experimental animal study was conducted at the Surgical Medical Research Institute and Katz Group Rexall Centre for research at the University of Alberta, between April 2016 and February 2017.

After induction of general inhalation anesthesia using isofluorane ( $2 \%$ in air; IA) to set up vein cannulation, anesthesia was maintained under one of the two anesthetic options followed by the second one (i.e TIVA followed by IA or vice versa) allowing a washout period to occur in between, during which the animal shows a positive toe pinch reflex.

\section{Study subjects}

A total of 30 Sprague-Dawley rats were approved for this study. All rats were housed in pairs within the housing facility of Health Sciences Laboratory Animal Services at the University of Alberta. Eight rats were used initially as a pilot study.

\section{Experimental procedure Preparation and anesthesia}

Pre-operatively, age, sex, and weight of the rats were documented and a unique identifying number was given. Rats were then placed in an induction chamber saturated with $2 \%$ isoflurane. Anesthesia was maintained using either inhaled isoflurane $1.5-5 \%$ or a combination of propofol (10 mg/ml and $40-50 \mathrm{mg} / \mathrm{kg} / \mathrm{h} \mathrm{IV}$ infusion) [18] and remifentanyl $(5 \mathrm{mcg} / \mathrm{ml}$ and $0.4 \mathrm{mcg} / \mathrm{kg} / \mathrm{min} \mathrm{IV}$ infusion) [19] after establishing IV access (either via tail or femoral vein). Ampicillin ( $50 \mathrm{mg} / \mathrm{kg} \mathrm{SC}$ ), meloxicam (1-2 $\mathrm{mg} / \mathrm{kg} \mathrm{SC})$ and ringer's lactate $(1 \mathrm{ml} / \mathrm{kg} / \mathrm{hr}$. intraperitoneal) were administered preoperatively. The rat was then transferred to the surgical table and placed on a restraining board with an integrated circulating fluid heating pad with temperature set at $37^{\circ} \mathrm{C}$. A respiratory belt (Kent Scientific Co., USA), a rectal thermometer probe and a vital signs monitoring foot sensor (STARR Life Sciences ${ }^{\oplus}$ Mouse $\mathrm{Ox}^{\circ}$ Plus) were attached. The depth of anesthesia was determined by eliciting a toe pinch reflex, observing the respiratory rate and pattern of breathing, and finally the tolerance and response to airway stimulation to endoscope insertion. If TIVA was used for maintenance, isoflurane concentration was reduced to $0.5 \%$, and then turned off after a period of five minutes. The depth of the anesthesia was then assessed again and the rate of infusion was adjusted accordingly. Baseline and periodic readings of heart and respiratory rate, peripheral capillary oxygen saturation ( $\mathrm{SpO} 2)$, temperature and mucous membrane color were all recorded every five minutes.

\section{Laryngoscopy and laryngeal EMG}

Once the rat was adequately anesthetized under either IA or TIVA, room air (21\% O2) was delivered through the nasal mask for 1-2 min to maintain $\mathrm{SpO}_{2}$ above $90 \%$. The rat was then positioned supine on the experimental workstation inside a Faraday cage. By retracting the tongue, the larynx was visualized and lidocaine $1 \%$ $(1.67 \mathrm{mg} / \mathrm{kg}$ ) was applied topically under telescopic guidance. A nebulizer was also connected to the nasal cone and lidocaine $1 \%$ was delivered for $\sim 1 \mathrm{~min}$. This step was aborted in future experiments after presumed lidocaine toxicity mortality was encountered.

\section{Laryngoscopy}

While the animal was spontaneously breathing, a zero degree $2.7 \mathrm{~mm}$ rod lens telescope (KARL-STORZ ${ }^{\circ}$, Germany) connected to an image capture unit, was used to visualize vocal cords' movements.

\section{Laryngeal electromyography}

Once the larynx was exposed, LEMG recordings from the posterior cricoarytenoid (PCA) muscle were obtained by 
inserting a monopolar needle electrode (29GA, $37 \mathrm{~mm}$ ) (Rochester Electro-Medical, USA) transorally under direct rigid endoscopic visualization with each anesthetic regimen. Since the PCA muscle is responsible for vocal cord abduction during inspiration, in addition to the ease of electrode insertion in comparison to other adductor intrinsic laryngeal muscles in the tenuous rat airway, we elected to choose it as our muscle of choice to obtain LEMG recordings. A ground electrode (27G, $12 \mathrm{~mm}$ ) (Ambu ${ }^{\circ}$ Neuroline Subdermal, Malaysia) was secured into the chest. Electrodes were connected to amplifiers (AM Systems, Carlsborg, WA) and activity was filtered between $300 \mathrm{~Hz}$ and $1 \mathrm{kHz}$, amplified at $\mathrm{x} 10 \mathrm{k}$ and sampled at $1 \mathrm{kHz}$ (Powerlab 16/30; AD Instruments, Colorado Springs, CO). A piezoelectric chest belt was connected to the recording system in order to detect chest wall movements and correlate between LEMG signal and the respiratory cycle. A minimum of ten respiratory cycles was digitally recorded from the muscle for off-line analysis.

\section{Recovery, postoperative care and euthanasia}

Upon the conclusion of the experiment the rat was transferred to a new cage to allow recovery from anesthesia. Each rat was housed individually for $2 \mathrm{~h}$ post-operatively to be monitored and assessed clinically every fifteen minutes. This included activity, response to external stimuli, appearance and feeding. The animal was then euthanized by decapitation under isoflurane anesthesia.

\section{Outcome measures}

\section{Primary outcomes: Proportion of complete rigid laryngoscopy and LEMG}

A successful experiment was defined as completion of both laryngeal endoscopy and the ability to obtain a reliable LEMG recording. A complete laryngoscopy was defined as a minimally interrupted, well-tolerated rigid endoscopy of the respiratory action of the larynx while the subject goes through ten cycles of spontaneous breathing. For LEMG, ten consecutive respiratory related bursts of activity were required and the mean amplitudes and burst durations of the LEMG signal were analyzed and calculated using Lab ChartPro8.

Criteria used to abort the experiment included: signs of hemodynamic instability encountered during intraoperative monitoring (persistent maximal scores of respiratory distress, i.e. apnea/hypopnea, or sustained heart rate deviations) or a ninety minutes maximum duration as a cut-off point to achieve the appropriate depth of anesthesia.

\section{Secondary outcomes: Mortality and morbidity}

Mortalities encountered were documented. Morbidities experienced were defined as respiratory events during the procedure, which included laryngeal spasm, apnea and hypopnea requiring interruption of the procedure.

\section{Statistical analysis}

Demographics were summarized as means, standard deviation $(\mathrm{SD})$, minimal and maximal values. Student t-test was used for comparing means, and 95\% confidence intervals were provided. Fisher's exact test and chi square were used to compare proportions of mortality and morbidity between anesthetic regimens [20].

Based on a previous study from our laboratory using only propofol as TIVA [18], a 70\% respiratory morbidity rate was demonstrated. A decision was made that a reduction to $15 \%$ would be statistically and clinically significant. Based on $16.67 \%$ mortality rate and accepting a $p$ value of 0.05 and a power of $80 \%$, the sample size would be ten per group. Allowing for unforeseen morbidity, five rats were added to each group for a total of 30 rats.

As part of the LEMG waveform evaluation, we included motor unit potential assessment in the form of amplitude and burst duration. The amplitude was calculated from the height, the burst duration from the period and 60 divided by the period to obtain the respiratory rate using peak analysis in Lab ChartPro8.

\section{Results}

A total of 30 rats were used. The mean age was $7.56 \pm$ 5.79 months $(3-18)$. Thirteen were males and seventeen were females. The mean weight was $509.02 \pm 258.24 \mathrm{~g}$ (245-1200). The basic clinical parameters for the two groups are described in Table 1. All 8 rats from the pilot group underwent TIVA and two of them underwent a successful experiment.

Seventeen (77.3\%) rats underwent both TIVA and IA, two $(9.1 \%)$ underwent IA only. Three (13.6\%) mortalities were encountered in total. Two of them occurred during anesthetic inhalational induction and one mortality was likely due to lidocaine toxicity while on IA. Eight rats (47.06\%) were maintained with TIVA first followed by IA and twelve (52.94\%) were maintained with IA first followed by TIVA. See Fig. 1.

All nineteen rats that underwent IA had a successful experiment requiring a maximum period of $15 \mathrm{~min}$, i.e. tolerated endoscopy without major respiratory events and completed a reproducible LEMG. Out of the 17 rats that underwent TIVA, nine of them (52.94\%) completed a successful experiment requiring a duration between 45 and $90 \mathrm{~min}$. See Fig. 2. The eight unsuccessful experiments $(47.06 \%)$ were mainly due to inability to achieve an appropriate anesthetic plane. Seven of these rats continued to be responsive and intolerant of endoscopy despite escalation of the TIVA dosage to as high as 3.5 times the weight and boluses, whereas one animal 
Table 1 Parameters of the rats

\begin{tabular}{lll}
\hline Parameter & TIVA $(n=17)$ & IA $(n=19)$ \\
\hline Age (months) Mean \pm SD (range) & $7.18 \pm 6.1(3-18)$ & $6.74 \pm 5.9(3-18)$ \\
Males & 9 & 9 \\
Females & 8 & 10 \\
Weight (gm) Mean \pm SD (range) & $514.88 \pm 219.28(245-980)$ & $488 \pm 217.95(245-980)$
\end{tabular}

developed bradycardia down to 70 beats per minute and the $\mathrm{SpO}_{2}$ dropped to $60 \%$, and the procedure was aborted to ensure safety. In the nine successful experiments under TIVA, no mortalities were encountered. However, two rats (11.76\%) developed apneic events for seconds during the procedure and recovered spontaneously.

While the rats were maintained on IA, no morbidities were encountered. Only one (5.26\%) mortality took place (presumably due to lidocaine toxicity). Comparing both anesthetic regimens, no statistical significance was evident for morbidity $(p=0.096)$ or mortality $(p=0.679)$, however, IA proved to be superior to TIVA in performing successful experiments $(p=0.0008)$.

With regards to LEMG variables, it was noted that the mean amplitude of LEMG in TIVA is $66.9 \%$ that of IA. However, there was no significant difference between mean amplitudes $-1.79 \pm 9.88 \mathrm{mV}(95 \% \mathrm{CI}-1.79-2.2, p=0.1985)$ or mean burst duration $0.27 \pm 0.75$ s $(95 \%$ CI $-0.23-0.76, p$ $=0.82605)$. See in the Additional file 1: Table S1 for details.

Two rats had the electrode maintained in the same position without manipulations while the anesthetic regimens were switched. No statistical difference in mean amplitude or mean burst duration was evident individually $(p>0.05)$ despite the amplitude being lower on TIVA. The PCA contraction displays a pre-inspiratory

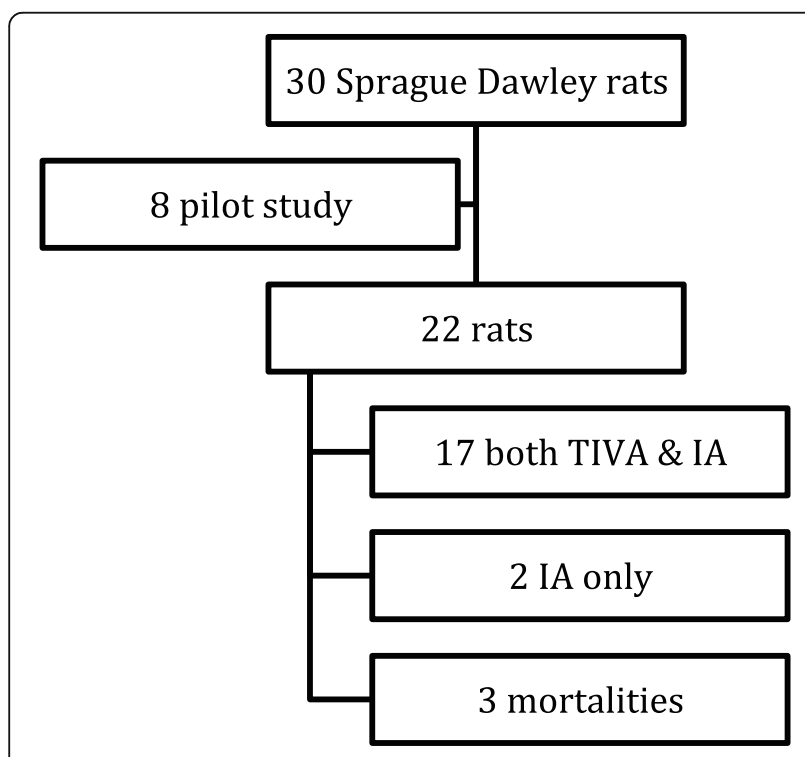

Fig. 1 General scheme of the study pattern of activity in both IA and TIVA consistently. See Fig. 3.

Of note, the time required to perform a full experiment using IA was $10-15 \mathrm{~min}$ compared to $45-90 \mathrm{~min}$ when using TIVA.

When comparing clinical parameters during the recovery period, no morbidities or mortalities occurred during recovery.

\section{Discussion}

This study compared the use of TIVA and IA during laryngoscopy and LEMG recording in rat models. Our endpoints were the ability to perform a complete endoscopy and neurophysiological recordings with the least morbidity and mortality. Our results show that TIVA is unlikely to be the anesthetic of choice for endoscopy and LEMG recordings in a rat model.

Balancing adequate depth of anesthesia and stability of spontaneous respiration during pediatric endoscopic surgery may be difficult to maintain using TIVA [21, 22]. Additionally, drug dosing appears more demanding with TIVA and higher infusion rates are sometimes necessary to provide the desired plasma concentrations [2]. Malik and Sen [23] reported 5.3\% respiratory related morbidity manifested as brief episodes of desaturation due to malposition of the airway and laryngospasm with intermittent TIVA for pediatric endoscopic procedures. On the other hand, evidence suggests that TIVA reduces airway reactivity; decreases bronchospasm and laryngospasm in children [4, 24].

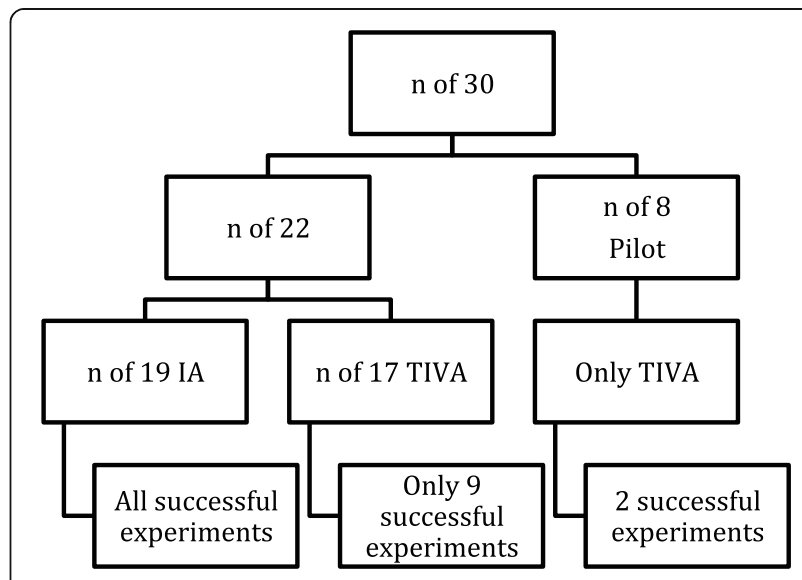

Fig. 2 Successful experiments under TIVA and IA 


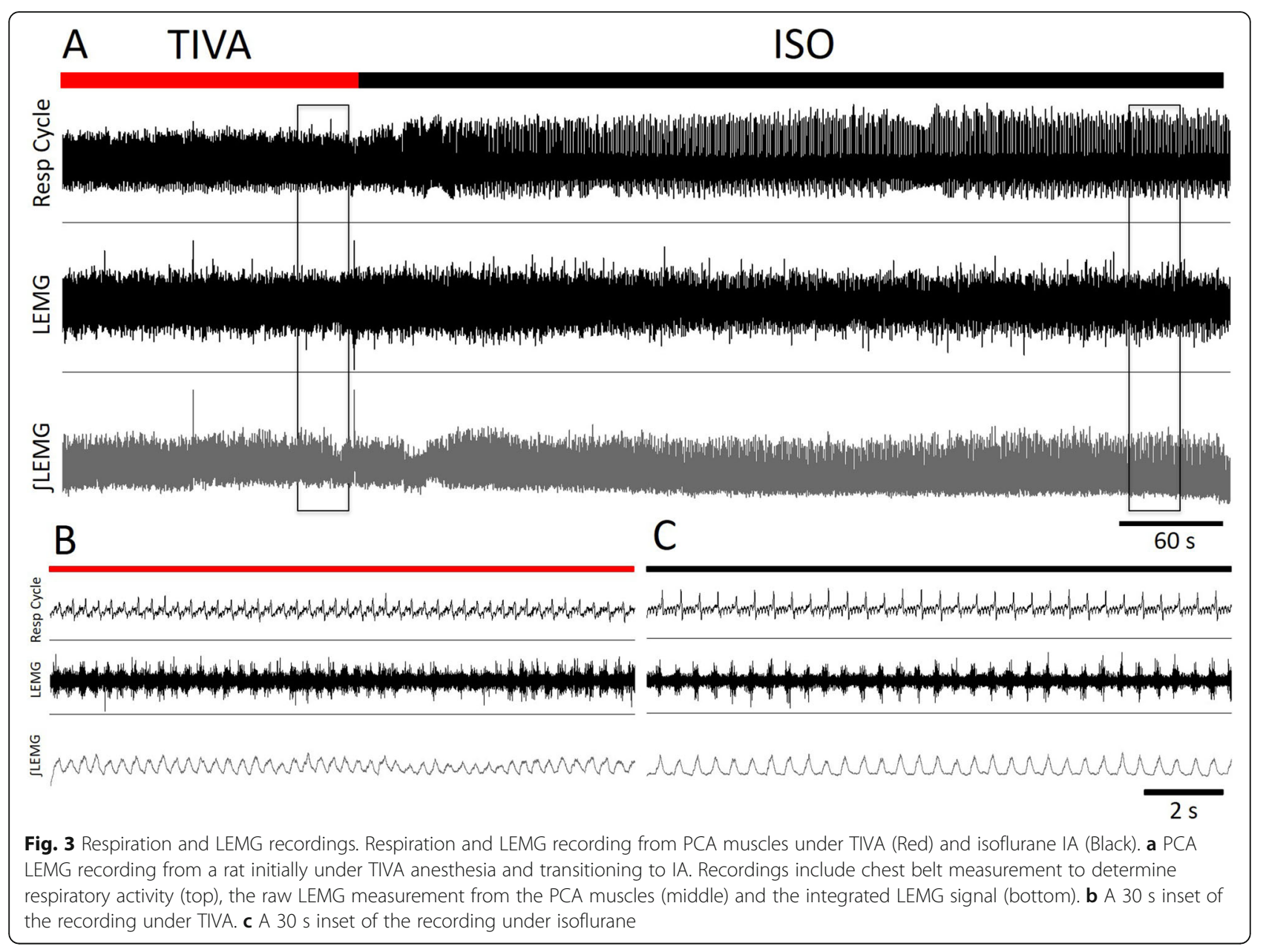

In the current study, the morbidity rate was reduced from $70 \%$ based on previous experiments performed by the senior author HE [18] to $11.7 \%$ which is considered clinically significant in the current experiment. This may be due to the effect of adding remifentanyl.

Several studies have compared different types of anesthetics regimes in pediatric otolaryngology surgeries. A direct comparison between TIVA (propofol plus remifentanil) and volatile anesthetics indicated that TIVA is superior for induction, maintenance and recovery from anesthesia in children undergoing flexible fiberoptic bronchoscopy, adenoidectomy and/or tonsillectomy [25, 26].

Evidence in pediatric IA suggests that it may cause apnea following induction, especially if great concentrations were delivered [2]. This may explain the unexpected mortalities encountered during induction under IA in our current study.

Several anesthetics in experimental animal models have been used to perform safe, interpretable and reliable endoscopy and/or LEMG, but direct comparisons and proof of reproducibility are limited [13-17, 27-32]. These included intraabdominal barbitone sodium [27], mixture of intramuscular ketamine hydrochloride and xylazine hydrochloride [14, 15, 30] intramuscular ketamine only [28], combination of inhaled isoflurane and intraperitoneal ketamine and xylazine [16, 17, 29, 31], intraperitoneal/ intravenous pentobarbital sodium [32], and isoflurane only [13]. With the exception of ketamine and isoflurane, none of these drugs are used in clinical practice. Interestingly, only scant reports on mortality and morbidity have been previously described given the fragility of the animal and its delicate airway, with prior experience indicating up to $20 \%$ mortality $[13,18,33,34]$.

Our results indicate that LEMG can be reliably performed under IA in a rat model. Despite previous studies in human spinal surgery [5-8] and animal studies [35] suggesting that the duration and concentration of IA affect evoked electromyogram parameters, specifically amplitude and latency, our results showed no difference in spontaneous LEMG variables between anesthetics. Several LEMG clinical studies [36-39] have used TIVA instead of IA when studying laryngeal disorders. The exact reasons are unclear, perhaps due to the increased use of TIVA among 
pediatric anesthetists or the potential effect of IA on LEMG.

One important observation in our study is the reduction in the duration of a complete experiment using IA (10-15 min) compared to TIVA (45-90 min). The advantages of using IA compared to TIVA in terms of reduction in induction time, maintenance of stable breathing, lack of laryngeal bronchospasm and emergence from anesthesia has been also reported previously in pediatric studies $[21,40]$. In our study, the longer duration required while on TIVA was mainly due to inability to achieve an appropriate anesthetic plane to perform the experiment. This may well be due to IV agents displaying excessive inter-individual variability to TIVA maintenance that cannot be easily estimated [2] or perhaps associated with the large body weight of some rats used for this study and the accumulation of fat mass that may alter the dose necessary to establish the optimal surgical plane.

Tsai and colleagues [41] compared the recovery from laryngoscopy procedures under propofol TIVA and conventional isoflurane in a canine model. The TIVA group was significantly better than the isoflurane group in terms of smoothness of recovery from surgery, defined as absence of struggling, vocalization, or excitement and requiring little or no physical restraint to prevent self-injury. However, the isoflurane group recovered faster from anesthetic. No significant difference was observed between the two groups in terms of adverse effects, which was comparable to our findings.

LEMG is considered a valuable clinical and research tool to assess different pathologies in laryngeal motor function. Its' use has been described in the literature for humans and in experimental models as an outcome measure following a specific laryngeal intervention. As part of the reporting practice, the type of anesthetic used throughout the procedure should be documented [42]. Proponents of LEMG argue its prognostic and diagnostic values that may guide treatment decisions in patients with vocal fold mobility disorders [37, 43].

Multiple intrinsic laryngeal muscles were described in the literature as a point for recording LEMG activity in both animals and humans. This includes thyroarytenoid [9-12, 15-18, 33, 36-39, 44-49], cricothyroid [44, 46, $48,50]$, PCA $[11-13,15,16,18,27,32,33,37,38,44$, $45,49,51,52]$ and lateral cricoarytenoid [15] either individually or in combination. The authors have selected the PCA muscle, as it's the single laryngeal muscle responsible for vocal fold abduction. PCA contraction has been pre-inspiratory on a consistent basis noted by the piezoelectric chest belt in our study.

One of the study limitations is the wide range of weight (245 g-980 g) and the large weight potentially contributing in the rat's morbidity and/or mortality while under general anesthesia. Despite the known typical weight of laboratory rats ranging between $300 \mathrm{~g}$ and $500 \mathrm{~g}$, mortalities encountered were amongst smaller animals weighing equal to or less than $400 \mathrm{~g}$ and morbidities were seen within the mean of weight.

We acknowledge that the monopolar needle electrode was inserted in different locations within the PCA muscle with each anesthetic regimen, which may have affected the LEMG recordings. We are aware that recordings depend on the size of muscle fibers, the proximity of the electrode to large muscles, and the depth of electrode insertion. However, maintaining the electrode in the same position is practically difficult to achieve. A proper washout period between the two anesthetic methods cannot be feasible. Still, we were able to keep the electrode in the same location without manipulation in 2 rats and found no statistical difference in mean amplitude or burst duration.

An additional limitation is the fact that the rats were not blindly randomized for the anesthetic regimens, nor were the results concealed due to the nature of the experiment. However, we used a cross over trial instead. In such case, randomization or which anesthetic was started first does not matter, as the rat will undergo both anesthetic regimens regardless. Also, TIVA and IA are known to have rapid onset/offset action, the effect of anesthetic is reversible, the period of administration is short; the condition is relatively stable as the rats were completely healthy, and the carry-over is not an issue [53]. This permitted convenience and efficiency of the project [54]. This design also allowed amplification of the sample size used.

In the future, we aim to replicate the same experiment in pediatric patients and compare anesthetic regimens and their effect on laryngoscopy and LEMG, as IA is a useful translational model for laryngoscopy and LEMG experiments.

\section{Conclusion}

Contrary to our hypothesis, IA did not affect the quality of LEMG and allowed a seamless rigid endoscopy in rat models superior to TIVA. It proved to be quick, easy and safe to administer. We conclude that this is a reliable translational model for laryngoscopy and LEMG experiments.

\section{Additional file}

Additional file 1: Table S1. LEMG parameters. (DOCX $61 \mathrm{~kb}$ )

\section{Abbreviations}

IA: Inhalational anesthesia; IV: Intravenous; kg/min: Kilogram/minute; L/ min: Liters/minute; LEMG: Laryngeal electromyography; mcg/kg/ min: Microgram/kilogram/minute; mcg/ml: Microgram/milliliter; mg/ kg: Milligram/kilogram; mg/ml: Milligram/milliliter; min: Minutes; ml: Milliliter; $\mathrm{ml} / \mathrm{kg} / \mathrm{hr}$ : Milliliter/kilogram/hour; mm: Millimeter; $\mathrm{O}_{2}$ : Oxygen; PCA: Posterior 
cricoarytenoid; SC: Subcutaneous; SD: Standard deviation; $\mathrm{SpO}_{2}$ : Oxygen saturation; TIVA: Total intravenous anesthesia

\section{Funding}

This study has been funded by the Edmonton Civic Employees Grant. Malak Jamal Gazzaz acknowledges her residency scholarship through Umm Al-Qura University, Makkah, Saudi Arabia. SP laboratory is funded by NSERC, CIHR, Canadian Lung Association and Women and Children Health Research Institute.

\section{Availability of data and materials}

The datasets during and/or analyzed during the current study are available from the corresponding author on reasonable request.

\section{Authors' contributions}

MJG contributed to the experimental procedures, data analysis and interpretation, and manuscript preparation. She has approved the final manuscript in its current form. JS contributed to the experimental procedures, data analysis and interpretation. She has approved the final manuscript in its current form. SP contributed to the experimental procedures, oversaw the technical aspects of the research and contributed to manuscript preparation. She has approved the final manuscript in its current form. BT contributed to the experiment conception and design and contributed to the pilot study. He has approved the final manuscript in its current form. CJ contributed to the conception and design of the study, and provided input to the manuscript. She has approved the final manuscript in its current form. HE was responsible for the conception, design, and overall execution of the study. He contributed to the manuscript preparation and has approved the final manuscript in its current form.

\section{Ethics approval and consent to participate}

Ethics approval was obtained from the University of Alberta Health Research Ethics Board, Canadian Council on Animal Care guidelines and policy Animal Care and Use Committee for health sciences and University of Alberta's Animal Welfare (Assurance Number: \#A5070-01) prior to study commencement.

\section{Consent for publication}

Not applicable.

\section{Competing interests}

The authors declare that they have no competing interests.

\section{Publisher's Note}

Springer Nature remains neutral with regard to jurisdictional claims in published maps and institutional affiliations.

\section{Author details}

'Division of Otolaryngology-Head and Neck Surgery, Department of Surgery, University of Alberta, 2C3.57 Walter MacKenzie Centre, Edmonton, AB T6G 2R7, Canada. ${ }^{2}$ Neuroscience and Mental Health Institute, University of Alberta, Edmonton, AB, Canada. ${ }^{3}$ Women and Children Research Institute, University of Alberta, Edmonton, AB, Canada. ${ }^{4}$ Department of Physiology, University of Alberta, Edmonton, AB, Canada. ${ }^{5}$ Stanford University Pediatric Regional Anesthesia, Stanford University, Stanford, California, USA.

Received: 8 April 2018 Accepted: 5 October 2018

\section{Published online: 20 October 2018}

\section{References}

1. Malherbe S, Whyte S, Singh P, Amari E, King A, Mark Ansermino J. Total intravenous anesthesia and spontaneous respiration for airway endoscopy in children - a prospective evaluation. Paediatr Anaesth. 2010;20:434-8.

2. Lerman J, JÖhr M. Inhalational anesthesia vs total intravenous anesthesia (TIVA) for pediatric anesthesia. Paediatr Anaesth. 2009;19:521-34.

3. McCormack JG. Total intravenous anaesthesia in children. Curr Anaesth Crit Care. 2008;19:309-14. https://doi.org/10.1016/j.cacc.2008.09.005.

4. Gaynor J, Ansermino JM. Paediatric total intravenous anaesthesia. BJA Educ. 2016;16:369-73.
5. Chen X, Xu L, Wang Y, Xu F, Du Y, Li J. Sevoflurane affects evoked electromyography monitoring in cerebral palsy. Open Med. 2016;11:138-42. https://doi.org/10.1515/med-2016-0027.

6. Banoub M, Tetzlaff JE, Schubert A. Pharmacologic and Physiologic Influences Affecting Sensory Evoked Potentials: implications for perioperative monitoring. Anesthesiology. 2003;(3):716-37.

7. Chong CT, Manninen P, Sivanaser V, Subramanyam R, Lu N, Venkatraghavan $\mathrm{L}$. Direct comparison of the effect of desflurane and sevoflurane on intraoperative motor-evoked potentials monitoring. J Neurosurg Anesthesiol. 2014;26:306-12.

8. Chen Z. The effects of isoflurane and propofol on intraoperative neurophysiological monitoring during spinal surgery. J Clin Monit Comput. 2004;18:303-8.

9. Gartlan MG, Peterson KL, Hoffman HT, Luschei ES, Smith RJH. Bipolar hooked-wire electromyographic technique in the evaluation of pediatric vocal cord paralysis. Ann Otol Rhinol Laryngol. 1993;102:695-700.

10. Wohl DL, Leshner RT, Kilpatrick JK, Shaia WT. Intraoperative pediatric laryngeal electromyography: experience and caveats with monopolar electrodes. Ann Otol Rhinol Laryngol. 2001;110:524-31.

11. Berkowitz RG. Laryngeal electromyography findings in idiopathic congenital bilateral vocal cord paralysis. Ann Otol Rhinol Laryngol. 1996;105:207-12.

12. Berkowitz RG, Ryan MM, Pilowsky PM. Respiration-related laryngeal electromyography in children with bilateral vocal fold paralysis. Ann Otol Rhinol Laryngol. 2009;118:791-5.

13. McRae BR, Kincaid JC, Illing EA, Hiatt KK, Hawkins JF, Halum SL. Local neurotoxins for prevention of laryngeal Synkinesis after recurrent laryngeal nerve injury. Ann Otol Rhinol Laryngol. 2009;118:887-93. https://doi.org/10. 1177/000348940911801210.

14. Tessema B, Roark RM, Pitman MJ, Weissbrod P, Sharma S, Schaefer SD. Observations of recurrent laryngeal nerve injury and recovery using a rat model. Laryngoscope. 2009;119:1644-51.

15. Pitman MJ, Weissbrod P, Roark R, Sharma S, Schaefer SD. Electromyographic and histologic evolution of the recurrent laryngeal nerve from transection and anastomosis to mature reinnervation. Laryngoscope. 2011;121:325-31.

16. Tessema B, Pitman MJ, Roark RM, Berzofsky C, Sharma S, Schaefer SD. Evaluation of functional recovery of recurrent larymgeal nerve using transoral laryngeal bibopar electromyography: a rat model. New York. 2008; 117:604-8.

17. Kupfer RA, Old MO, Oh SS, Feldman EL, Hogikyan ND. Spontaneous laryngeal reinnervation following chronic recurrent laryngeal nerve injury. Laryngoscope. 2013;123:2216-27. https://doi.org/10.1002/lary.24049.

18. Jomah MA, Jomah MA. Does botulinum toxin type a alter the consequences of recurrent laryngeal nerve transection in the rat model ? By master of science in experimental Surgery University of Alberta. 2014.

19. Ismaiel NM, Chankalal R, Zhou J, Henzler D. Using remifentanil in mechanically ventilated rats to provide continuous analgosedation. J Am Assoc Lab Anim Sci. 2012;51:58-62.

20. SISA. Simple Interactive Statistical Analysis. http://www.quantitativeskills. com/sisa/index.htm. Accessed 10 Feb 2018.

21. Xu J, Yao Z, Li S, Chen L. A non-tracheal intubation (tubeless) anesthetic technique with spontaneous respiration for upper airway surgery. Clin Investig Med. 2013;36:151-7.

22. Dilos BM. Anesthesia for pediatric airway endoscopy and upper gastrointestinal endoscopy. Int Anesthesiol Clin. 2009;47:55-62.

23. Malik M, Sen S. Propofol anesthesia is an effective and safe strategy for pediatric endoscopy [4]. Paediatr Anaesth. 2006;16:220-1.

24. Lauder GR. Total intravenous anesthesia will supercede inhalational anesthesia in pediatric anesthetic practice. Paediatr Anaesth. 2015;25:52-64.

25. Chen $L, Y u L$, Fan $Y$, Manyande A. A comparison between total intravenous anaesthesia using propofol plus remifentanil and volatile induction/maintenance of anaesthesia using sevoflurane in children undergoing flexible fibreoptic bronchoscopy. Anaesth Intensive Care. 2013:41:742-9.

26. Grundmann U, Uth M, Eichner A, Wilhelm W, Larsen R. Total intravenous anaesthesia with propofol and remifentanil in paediatric patients: a comparison with a desflurane-nitrous oxide inhalation anaesthesia. Acta Anaesthesiol Scand. 1998:42:845-50. https://doi.org/10.1111/j.1399-6576. 1998.tb05332.x.

27. Liu HJ, Dong MM, Chi FL. Functional remobilization evaluation of the paralyzed vocal cord by end-to-side neurorrhaphy in rats. Laryngoscope. 2005; 115:1418-20 
28. Motoyoshi K, Hyodo M, Yamagata T, Gyo K. Restoring vocal fold movement after transection and immediate suturing of the recurrent laryngeal nerve with local application of basic fibroblast growth factor: an experimental study in the rat. Laryngoscope. 2004;114:1247-52.

29. Rubin AD, Hogikyan ND, Oh A, Feldman EL. Potential for promoting recurrent laryngeal nerve regeneration by remote delivery of viral gene therapy. Laryngoscope. 2012;122:349-55.

30. Shiotani A, Nakagawa H, Flint PW. Modulation of myosin heavy chains in rat laryngeal muscle. Laryngoscope. 2001;111:472-7. https://doi.org/10.1097/ 00005537-200103000-00017.

31. Old MO, Oh SS, Feldman E, Hogikyan ND. Novel model to assess laryngeal function, innervation, and reinnervation. Ann Otol Rhinol Laryngol. 2011;120:331-8.

32. Berkowitz RG, Chalmers J, Sun QIJ, Pilowsky PM. Respiratory activity of the rat posterior cricoarytenoid muscle. Ann Otol Rhinol Laryngol. 1997;106: 897-901.

33. Xu W, Han D, Hu I, Fan E. Characteristics of experimental recurrent laryngeal nerve surgical injury in dogs. Ann Otol Rhinol Laryngol. 2009;118:575-80.

34. Sakowski SA, Heavener SB, Lunn JS, Fung K, Oh SS, Spratt SK, et al. Neuroprotection using gene therapy to induce vascular endothelial growth factor-a expression. Gene Ther. 2009;16:1292-9.

35. Haghighi SS, Madsen R, Green KD, Oro JJ, Kracke GR. Suppression of motor evoked potentials by inhalation anesthetics. J Neurosurg Anesthesiol. 1990;2:73-8.

36. Scott A, Chong P, Randolph G, Hartnick C. Intraoperative laryngeal electromyography in children with vocal fold immobility: a simplified technique. Int J Pediatr Otorhinolaryngol. 2008;72:31-40.

37. AlQudehy Z, Norton J, El-Hakim H. Electromyography in children's laryngeal mobility disorders: a proposed grading system. Arch Otolaryngol - Head Neck Surg. 2012;138:936-41.

38. Jacobs IN, Finkel RS. Laryngeal electromyography in the management of vocal cord mobility problems in children. Laryngoscope. 2002;112:1243-8.

39. SC M, Braun N, DJ B, Chong P, JE K, CJ H. Intraoperative laryngeal electromyography in children with vocal fold immobility: results of a multicenter longitudinal study. Arch Otolaryngol Neck Surg. 2011;137:12517. https://doi.org/10.1001/archoto.2011.184.

40. Liao R, Li JY, Liu GY. Comparison of sevoflurane volatile induction/ maintenance anaesthesia and propofol-remifentanil total intravenous anaesthesia for rigid bronchoscopy under spontaneous breathing for tracheal/bronchial foreign body removal in children. Eur J Anaesthesiol. 2010;27:930-4

41. Tsai Y-C, Wang L-Y, Yeh L-S. Clinical comparison of recovery from total intravenous anesthesia with propofol and inhalation anesthesia with isoflurane in dogs. J Vet Med Sci. 2007;69:1179-82.

42. Blitzer A, Crumley RL, Dailey SH, Ford CN, Floeter MK, Hillel AD, et al. Recommendations of the Neurolaryngology study group on laryngeal electromyography. Otolaryngol - Head Neck Surg. 2009;140:782-793.e6. https://doi.org/10.1016/j.otohns.2009.01.026.

43. Munin MC, Heman-Ackah YD, Rosen CA, Sulica L, Maronian N, Mandel S, et al. Consensus statement: using laryngeal electromyography for the diagnosis and treatment of vocal cord paralysis. Muscle Nerve. 2016;53:850-5.

44. García-López I, Santiago-Pérez S, Peñarrocha-Teres J, del Palacio AJ, Gavilan J. Laryngeal Electromyography in Diagnosis and Treatment of Voice Disorders. Acta Otorrinolaringol. 2012;63:458-64. https://doi.org/10.1016/j. otoeng.2012.11.008.

45. Mu L, Yang S. An experimental study on the laryngeal electromyography and visual observations in varying types of surgical injuries to the unilateral recurrent laryngeal nerve in the neck. Laryngoscope. 1991;101(7 Pt 1):699-708.

46. Park HS, Jung SY, Yoo JH, Park HJ, Lee CH, Kim HS, et al. Clinical usefulness of ultrasonography-guided laryngeal electromyography. J Voice. 2016;30: 100-3. https://doi.org/10.1016/j.jvoice.2015.03.009.

47. Paniello RC, West SE, Lee P. Laryngeal Reinnervation with the hypoglossal nerve. Ann Otol Rhinol Laryngol. 2001;110:532-42.

48. Kianicka I, Diaz V, Renolleau S, Canet E, Praud JP. Laryngeal and abdominal muscle electrical activity during periodic breathing in nonsedated lambs. J Appl Physiol. 1998;84:669-75.

49. Watts TL, Wozniak JA, Davenport PW, Hutchison AA. Laryngeal and diaphragmatic activities with a single expiratory load in newborn lambs. Respir Physiol. 1997;107:27-35.
50. Rex MA. Laryngeal activity during the induction of anaesthesia in the cat. Aust Vet J. 1973;49:365-8.

51. Carlo WA, Kosch PC, Bruce EN, Strohl KP, Martin RJ. Control of laryngeal muscle activity in preterm infants. Pediatr Res. 1987;22:87-91.

52. Bliss MR, Wark H, McDonnall D, Smith ME. Functional electrical stimulation of the feline larynx with a flexible ribbon electrode array. Ann Otol Rhinol Laryngol. 2016;125:130-6.

53. Senn S, Ezzet F. Clinical cross-over trials in phase I. Stat Methods Med Res. 1999:8:263-78.

54. Lui K-J. Notes on crossover design. Enliven Biostat Metr. 2015;1:1-2.
Ready to submit your research? Choose BMC and benefit from:

- fast, convenient online submission

- thorough peer review by experienced researchers in your field

- rapid publication on acceptance

- support for research data, including large and complex data types

- gold Open Access which fosters wider collaboration and increased citations

- maximum visibility for your research: over $100 \mathrm{M}$ website views per year

At BMC, research is always in progress.

Learn more biomedcentral.com/submissions 